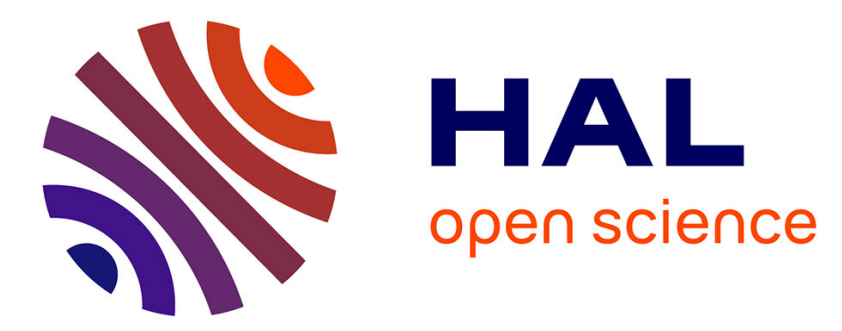

\title{
Lipoprotein-apheresis in familial hypercholesterolemia: Long-term patient compliance in a French cohort
}

Sophie Béliard, Antonio Gallo, Emilie Duchene, Alain Carrié, Randa Bittar, M. John John Chapman, Samir Saheb

\section{- To cite this version:}

Sophie Béliard, Antonio Gallo, Emilie Duchene, Alain Carrié, Randa Bittar, et al.. Lipoproteinapheresis in familial hypercholesterolemia: Long-term patient compliance in a French cohort. Atherosclerosis, 2018, 277, pp.66 - 71. 10.1016/j.atherosclerosis.2018.08.007 . hal-01909377

\section{HAL Id: hal-01909377 \\ https: / hal.sorbonne-universite.fr/hal-01909377}

Submitted on 31 Oct 2018

HAL is a multi-disciplinary open access archive for the deposit and dissemination of scientific research documents, whether they are published or not. The documents may come from teaching and research institutions in France or abroad, or from public or private research centers.
L'archive ouverte pluridisciplinaire HAL, est destinée au dépôt et à la diffusion de documents scientifiques de niveau recherche, publiés ou non, émanant des établissements d'enseignement et de recherche français ou étrangers, des laboratoires publics ou privés. 


\title{
Lipoprotein-apheresis in familial hypercholesterolemia: Long-term patient compliance in a French cohort
}

\author{
Sophie Beliard ${ }^{\mathrm{a}, 1,2}$, Antonio Gallo ${ }^{\mathrm{a}, \mathrm{b}, 2, *}$, Emilie Duchêne ${ }^{\mathrm{a}, \mathrm{b}}$, Alain Carriée,c, Randa Bittar ${ }^{\mathrm{d}}$, \\ M. John Chapman ${ }^{\mathrm{b}}$, Eric Bruckert ${ }^{\mathrm{a}, \mathrm{b}}$, Samir Saheb ${ }^{\mathrm{a}, \mathrm{b}}$

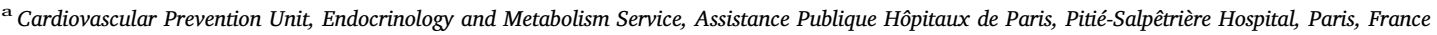 \\ ${ }^{\mathrm{b}}$ Sorbonne Universite, Inserm, Institute of Cardiometabolism and Nutrition (ICAN), UMR_S1166, Hôpital de la Pitié, Paris, France \\ ${ }^{c}$ Assistance publique-Hôpitaux de Paris (APHP), Hôpitaux Universitaires Pitié-Salpêtrière/Charles-Foix, Department of Biochemistry for Endocrinology and Oncology, \\ Obesity and Dyslipidemia Genetics Unit, France \\ d Biochemistry functional Unit for Metabolic Disease Assistance Publique/Hôpitaux de Paris, Groupe Hospitalier Pitié-Salpêtrière, Paris, France
}

H I G H L I G H T S

- An as high as $87.5 \%$ compliance with Lipoprotein Apheresis (LA) is found in severe FH.

- Main causes of missed procedure are holidays and/or concomitant illness.

- The frequency of treatment is the only determinant of low-compliance.

- Patients having more than $2 \mathrm{LA} /$ month have a 3-fold increased risk of low-compliance.

- Cardiovascular history and genotype are not associated with low-compliance.

\section{A R T I C L E I N F O}

\section{Keywords:}

Compliance

Lipoprotein apheresis

Familial hypercholesterolemia

Coronary heart disease

Dyslipidaemia

\begin{abstract}
A B S T R A C T
Background and aims: Lipoprotein apheresis (LA) is a complex therapeutic option and poor compliance can adversely affect treatment outcome. The aim of this study was to describe long-term compliance to treatment in patients undergoing regular LA therapy and to investigate factors related to low compliance.

Methods: We analysed 11,391 prescribed procedures of LA performed between 1990 and 2007 in 51 patients with familial hypercholesterolemia. Regular LA treatment was initiated in patients presenting with either homozygous familial hypercholesterolemia $(n=21)$, or severe heterozygous familial hypercholesterolemia ( $n=30$ ) with elevated LDL-cholesterol levels and who did not respond adequately to diet and drug therapy; the majority of these patients $(n=30)$ had cardiovascular disease at initiation of therapy.

Results: The overall observed compliance rate based on the number of achieved/programmed procedures was 87.5\%. Neither cardiovascular history nor subtypes of hypercholesterolemia was associated with compliance. In addition, there was no impact of patient demography on compliance. Treatment frequency alone significantly impacted non-compliance (i.e. patient with weekly procedures were less compliant). Interestingly, a non-significant decrease in compliance was observed among patients aged $<20$ years.

Conclusions: Despite the complexity of the LA procedure and its impact on the organisation of patients' daily lives, overall compliance was very high. The choice of an appropriate and adequate frequency of treatment significantly impacted patient compliance.
\end{abstract}

\section{Introduction}

Familial hypercholesterolemia (FH) is an autosomal dominant genetic disorder, resulting from mutations in genes encoding the lowdensity lipoprotein (LDL)-receptor, the apolipoprotein B (Apo-B), and the pro-protein convertase subtilisin/kexin 9 (PCSK9) [1]. The reduced

\footnotetext{
* Corresponding author. Unité de Prévention Cardiovasculaire, Service d'Endocrinologie Métabolisme, Groupe Hospitalier Pitié-Salpêtrière, Assistance Publique Hôpitaux de Paris, 83, boulevard de l'Hôpital, 75651, Paris Cedex 13, France.

E-mail address: antonio.gallo@aphp.fr (A. Gallo).

${ }^{1}$ C2VN, INRA, INSERM, Aix-Marseille Univ, Marseille, France and Endocrinology department, APHM, Marseille, France.

2 These authors contributed equally to this work.
} 
number or the absence of functional LDL-receptors on hepatocytes, results in a decreased uptake of LDL-cholesterol (LDL-C) particles from the plasma leading to elevated plasma LDL-C concentrations. In FH, the lifelong arterial exposure to elevated LDL-C results in cholesterol accumulation in arterial wall leading to premature atherosclerosis and cardiovascular events [2]. The heterozygous form of familial hypercholesterolemia (heFH) is one of the most frequent genetically-determined metabolic disorders in the general population with an estimated prevalence between $1 / 200$ and $1 / 250$ [3]. Without lipidlowering therapy, patients with heFH, have a 13-fold increase in cardiovascular risk [4]. However, even in the statin era, cardiovascular (CV) mortality rates remain more than double in heFH patients as compared to those in patients without FH [5]. Homozygous hypercholesterolemia (hoFH) is rare $(1 / 160,000$ to $1 / 300,000)$ but severe $[6,7]$. Indeed, children with hoFH develop premature CV in the first or second decade of life and often die suddenly before 30 years in the absence of treatment.

Familial hypercholesterolemia is characterised by high baseline LDL-C concentrations and a significant proportion of patients with severe $\mathrm{FH}$ does not reach treatment goals, remaining at increased risk for cardiovascular disease (CVD) [5,8]. The available drugs are often insufficient to achieve the recommended LDL-C goal, which makes the medical management of severe cases of FH challenging. Lipoprotein apheresis (LA) was developed as a rescue therapy for the treatment of severe refractory FH $[9,10]$. Current guidelines recommend LA treatment, where available, for the large majority of homozygous FH patients, which should be started by the age of 5 and no later than 8 years [11-13]. In France, LA is recommended in heFH patients when LDL-C levels remain very high despite maximum tolerated lipid lowering therapy, ie. $>300 \mathrm{mg} / \mathrm{dL}$ in primary prevention or $>200 \mathrm{mg} / \mathrm{dL}$ in secondary prevention [14].

LA is a complex therapeutic option and poor compliance can adversely affect treatment outcome. Few data are available on long-term compliance with LA.

The aim of the present study was to evaluate the long-term compliance of regular apheresis therapy in a large cohort of patients with severe $\mathrm{FH}$.

\section{Materials and methods}

In the present study, we analysed 11,391 LA procedures performed between January 1990 and December 2007 in fifty-one patients displaying severe $\mathrm{FH}$ who underwent LA in the Apheresis Centre at the Pitié-Salpêtrière University Hospital. Diagnosis of FH was made by genetic analysis or clinical criteria (the DLCN score for heFH and the EAS criteria for hoFH).

The apheresis method was selected according to age, pre-apheresis LDL-C levels, individual medical history, duration of treatment and history of allergy. Each patient was monitored (heart rate, blood pressure and $\mathrm{O}_{2}$ saturation monitoring) during the entire procedure. Levels of plasma total cholesterol (TC), lipoprotein (a) (Lp(a)), high-density lipoprotein-cholesterol (HDL-C) and triglycerides were measured and LDL-C was calculated according to Friedwald formula, before and immediately after each session for each patient.

During LA treatment, standard heparin (Heparin Immuno, BaxterImmuno, Vienna, Austria) or a combination of heparin and acid citrate dextrose (Whole blood system; ACDA, Baxter, Munich, Germany) was used for systemic anticoagulation. The treatments were performed using a peripheral venous vascular access (occasionally via an arteriovenous fistula) at a blood flow rate of $50-80 \mathrm{ml} / \mathrm{min}$. The blood volume to be treated was individually calculated for each patient.

The frequency of sessions was determined for each patient as a function of genetic profile and the evolution of plasma LDL-C levels between treatments; sessions were typically scheduled every 2-3 weeks, or every 7-10 days in patients displaying a more severe phenotype. Compliance was calculated as the percentage ratio of actually performed procedures on the theoretical number of expected procedures per patient.

The reasons for lack of compliance were collected from patients' files.

Informed consent was obtained from each patient included in the study and the study was performed in accordance with the recommendations of the Declaration of Helsinki.

\subsection{Statistical analysis}

Data are reported as mean \pm standard deviation (SD) for continuous parametric and median (Interquartile Range-IQR) for continuous non-parametric variables, and as frequency (percentage) for categorical variables. Normality of continuous variables distribution was tested using Kolmogorov-Smirnov test. Differences between groups were evaluated by ANOVA for parametric variables, Mann-Whitney for non-parametric variables. Distribution of categorical variables between groups was evaluated using the $\chi^{2}$ test. Correlations between two variables were assessed using a linear regression model and Pearson's correlation coefficient (r) was provided. For the multivariate logistic regression analysis, study population was stratified according to median compliance $(88 \%)$ and classified as compliant/non-compliant

Table 1

Main characteristics of study population, stratified according to genotype.

\begin{tabular}{|c|c|c|c|}
\hline & Whole population $(\mathrm{n}=51)$ & HoFH $(\mathrm{n}=21)$ & $\mathrm{HeFH}(\mathrm{n}=30)$ \\
\hline Age, years & $41.4(24.6-58.3)$ & $25.2(19.3-28.5)$ & $57.0(45.4-62.2)^{\mathrm{a}}$ \\
\hline Gender, males n (\%) & $27(52.9)$ & $9(42.9)$ & $18(60)^{\mathrm{a}}$ \\
\hline \multicolumn{4}{|l|}{ FH and LLT history } \\
\hline Age at LA initiation, years & $23.6(11.4-49.1)$ & $13.5(5.6-19.4)$ & $46.3(35.3-53.0)^{\mathrm{a}}$ \\
\hline LA frequency, $\mathrm{n}$ & $1.9 /$ month & $2.4 /$ month & $1.5 /$ month \\
\hline Follow-up duration, years & $9.52 \pm 5.4$ & $11.4 \pm 5.9$ & $8.0 \pm 4.5$ \\
\hline \multicolumn{4}{|l|}{ Cardiovascular events } \\
\hline Overall, n (\%) & $30(58.8)$ & $10(47.6)$ & $19(63.3)$ \\
\hline Coronary artery disease, $\mathrm{n}(\%)$ & 27 (52.9) & $8(38.1)$ & $19(63.3)^{\mathrm{a}}$ \\
\hline Carotid artery disease, $\mathrm{n}(\%)$ & $3(5.8)$ & $2(9.5)$ & $1(3.3)$ \\
\hline Peripheral artery disease, $n(\%)$ & $2(3.9)$ & $1(4.8)$ & $1(3.3)$ \\
\hline Aortic valve replacement, $\mathrm{n}(\%)$ & $6(11.7)$ & $4(19)$ & $2(6.7)$ \\
\hline
\end{tabular}

HoFH, homozygous familial hypercholesterolemia; HeFH, heterozygous familial hypercholesterolemia; LLT, lipid lowering treatment; LA, lipoprotein-apheresis; LDLc, low density lipoprotein-cholesterol.

${ }^{\mathrm{a}} p<0.05$ versus HoFH. 
Table 2

Lipid profile before and after lipoprotein apheresis.

\begin{tabular}{lllll}
\hline & Pre-LA & Post-LA & \% Acute reduction & \% Interval mean \\
\hline LDL-C, mg/dL & $223.3 \pm 25.6$ & $53.1 \pm 8.9$ & $(-76 \%)$ & $(-21 \%)$ \\
Triglycerides, mg/dL & $81.8 \pm 10.3$ & $29.2 \pm 4.5$ & $(-64 \%)$ & $(-17.4 \%)$ \\
HDL-C, mg/dL & $39.0 \pm 3.0$ & $33.2 \pm 2.8$ & $(-15 \%)$ & $(-4 \%)$
\end{tabular}

LA, lipoprotein apheresis; LDL-C, low-density-lipoprotein cholesterol; HDL-C, high-density lipoprotein cholesterol.

Interval means were calculated according to the following formula: $0.73\left(\mathrm{Lipid}_{\text {pre}}-\mathrm{Lipid}_{\text {post }}\right)+\operatorname{Lipid}_{\text {post }}[15]$.

accordingly. Compliance was further studied for its associations with CVD, frequency of LA, age at the beginning of treatment, genotype, sex (male $=1$ ) and physical distance from the apheresis centre. Statistical analysis was performed using SAS ${ }^{\oplus}$ software and a $p$ value $<0.05$ was considered significant.

\section{Results}

\subsection{Study population}

The demographic characteristics of the study population are summarized in Table 1.

Apart from LA, all patients were on maximal tolerated lipid-lowering treatment (LLT), consisting of a high-intensity statin plus ezetimibe (added after commercialization in 2005). Only one heFH patient was not on statin therapy because of documented muscular intolerance.

Patients were treated with one of the following procedures: 24 patients by the DALI ${ }^{\circ}$-System, 24 patients by the Liposorber ${ }^{\circ}$-System and 3 patients by Double Filtration Plasma Pheresis (DFPP). The duration per treatment session varied from 2 to $4 \mathrm{~h}$, according to the LA technique, to the volume of plasma processed and the rate of blood flow.

The mean \% reductions in TC, LDL-C, triglycerides and HDL-C in all patients after a single LA procedure are reported in Table 2 . The mean LDL-C after LA therapy was $53 \mathrm{mg} / \mathrm{dl}$, corresponding to a $76 \%$ reduction, similar to that of ApoB ( $36.4 \mathrm{mg} / \mathrm{dl}$ post-LA, $-75 \%)$; a higher than $50 \%$ acute reduction was also observed for triglycerides and Lp(a) $(29.2 \mathrm{mg} / \mathrm{dl}$ and $10.5 \mathrm{mg} / \mathrm{dl}$ post-LA, respectively) and a small decrease (15\%) was equally observed in HDL-C.

The mean decrease in LDL-C under lipid-lowering drug therapy in the hoFH patient group was $10-20 \%$ and $30-50 \%$ in heFH patients. A further 25-35\% LDL-C reduction was obtained when regular treatment with LA was introduced. Such reductions in LDL-C were maintained over the long-term; further reduction in LDL-C (10-15\%) was obtained by addition of ezetimibe.

\subsection{LDL-apheresis compliance}

Overall, 11,391 LA procedures were accomplished out of an expected total of 13,021 over a period of 18 years, leading to a treatment compliance of $87.5 \%$. In the whole population, 41 out of 51 patients (80.4\%) had an adherence to the LA procedure above $80 \%$, attaining $95 \%$ for 8 patients (16\%) of the cohort (Fig. 1). Almost $20 \%$ of patients $(\mathrm{n}=10)$ displayed poor compliance $(<80 \%)$.

Main causes of lack of compliance are shown in Fig. 2. The primary reasons for missed procedures were holidays and current illness or hospitalisation (31\%), whereas $22 \%$ were unspecified. No differences in the reason for missed procedure were observed between hoFH and heFH patients.

Univariate analysis did not show any influence of previous CVD, genotype, gender and distance from the centre on the overall compliance.

Multivariate regression analysis showed that a decreased compliance with LA was associated with the frequency of LA procedures/ month: patients undergoing more than $2 \mathrm{LA}$ /month had a 3-fold increased risk of being low-compliant (Table 3). These associations were independent of age at first LA, CVD, genotype, gender and distance from the apheresis centre.

During the median 11.4 years follow-up, 4 patients died of major cardiovascular complications ( 3 hoFH, 1 heFH) and 2 patients ( 1 hoFH, $1 \mathrm{heFH}$ ) died of non-cardiovascular causes. Five patients suffered an event after the beginning of LA, 3 undergoing coronary angioplasty, 1 femoral stenting for peripheral artery disease and 1 left carotid thrombendarterectomy. Sixteen patients quitted the LA service, of which 12 continued LA treatment elsewhere and 4 were lost to followup.

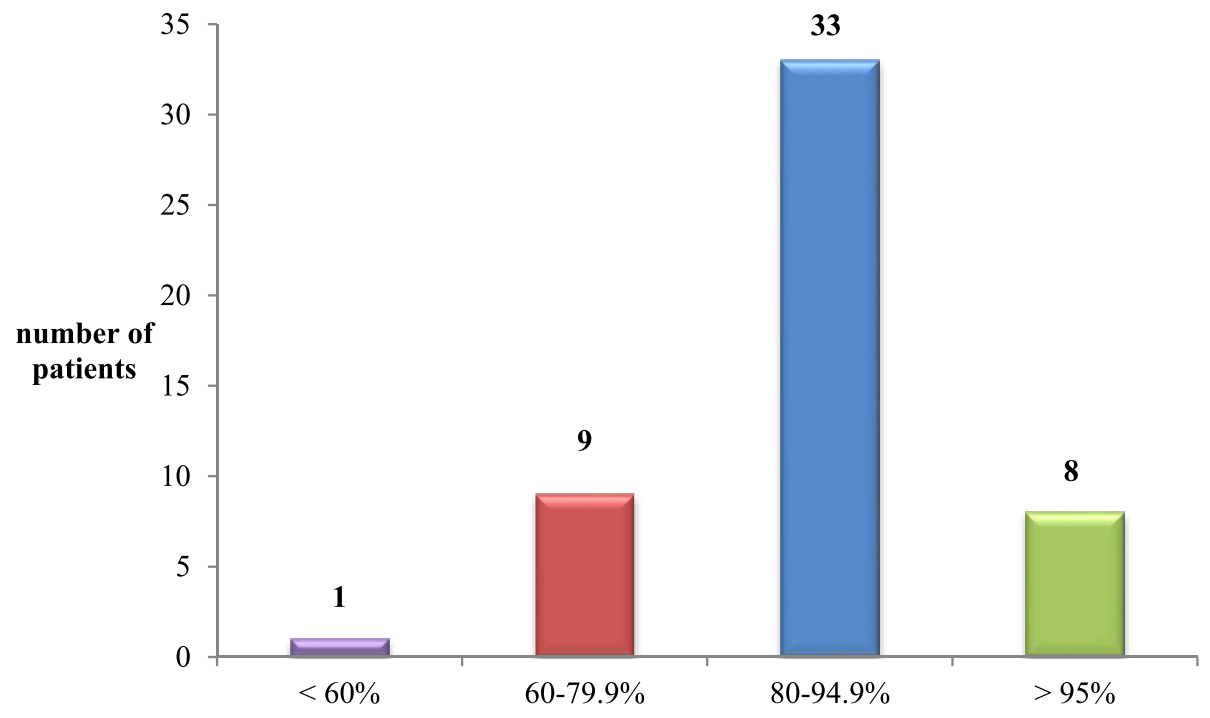

Fig. 1. Distribution of lipoprotein apheresis compliance (\%) in the study population. 


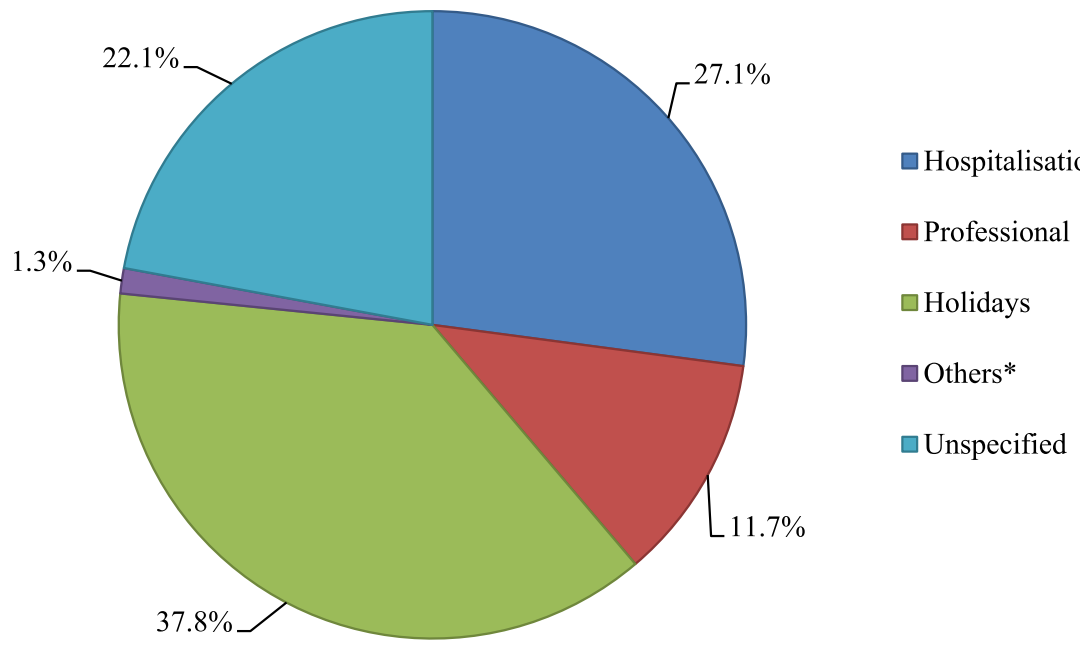

* Missed transport, mistaken appointment

Fig. 2. Main causes of low compliance (\%).

Table 3

Determinants of low adherence to LA in the study population by multivariate regression analysis.

\begin{tabular}{lll}
\hline Dependent variable & Low compliance & \\
\hline Independent variable & Multivariate ORs (95\% CI) & $p$ value \\
\hline Intercept & $0.145(0.015-1.42)$ & 0.0974 \\
Age at first LA & $1.051(0.983-1.124)$ & 0.1461 \\
Sex (male $=1)$ & $1.073(0.515-2.235)$ & 0.8503 \\
CVD (yes $=1)$ & $0.893(0.412-1.936)$ & 0.7743 \\
Genotype (heFH $=1)$ & $1.528(0.546-4.277)$ & 0.4196 \\
Frequency $(>2$ LA/month $=1)$ & $3.594(1.209-10.68)$ & 0.0213 \\
Distance $(<1 \mathrm{~h}$ drive $=1)$ & $0.919(0.472-1.792)$ & 0.8049 \\
\hline
\end{tabular}

Low compliance was defined as LA compliance lower than 88\% (median LA compliance).

LA, lipoprotein-apheresis; CVD, cardiovascular disease.

\section{Discussion}

By analysing more than 10,000 LA procedures over 9.5 years in a cohort of 51 severe FH patients, we observed a high treatment adherence. Finally, the only significant factor associated with a low compliance was the frequency of LA procedures per month.

There are multiple reasons that can explain the high level of compliance. The first is patient-related: the awareness of the severity of the disease and familial involvement, notably the personal or family history of cardiac events may contribute to increase the motivation towards regular treatments. A second reason may arise from the fact that assistance-related: "door-to-needle" transport costs are fully covered by the French social insurance. This is in contrast with countries where financial reasons may explain treatment discontinuation [2]. A degree of flexibility in the LA agenda allows patients who cancel an appointment to obtain a new one in one to three days. Moreover, a trained nursing team is dedicated to these patients: films and cartoons are proposed to children to entertain them throughout the procedure. The same nurse looks after the same young patient over time to develop a personal relationship. A third reason is procedure-related: only a small number of mild side effects has been reported during LA treatments. Potential adverse reactions that have been associated with LA include, but may not be limited to, hypotension, nausea, abdominal discomfort, tingling, chest pain, vasovagal reaction and prolonged bleeding from anticoagulant. Anaemia, resulting from the procedure or from blood sampling, is also a risk which may be minimized by iron supplementation [14,16-18]. Severe, but very rare, adverse events including an anaphylactic-like reaction have been reported in patients who were concomitantly treated with ACE-inhibitors [19]. In our cohort, the basic hemodynamic parameters are continuously monitored during the apheresis and the nursing team is trained to recognize any early signs of a bradykinin reaction, thus efficiently preventing the onset of any severe reaction.

Interestingly, despite these observations, a lower adherence was observed in patients with more than 2 LA procedures per month. The frequency of LA was the only significant factor affecting compliance. Only $78 \%$ of treatments were accomplished when sessions were performed weekly while $92 \%$ were achieved when LA was prescribed every three weeks. The treatment by LA is cumbersome and has incontestable consequences on the quality of life. Patients must organize one entire day consecrated to the procedures (including transport), and it is no surprise that the higher the frequency of procedures the lower the compliance. We analysed other predicting factors for non-compliance to LA. Surprisingly, we did not find any influence of age on the adherence to the LA treatment even in teenagers. We ranked patients by age categories and found that the 16-20-year-old patients had a $78 \%$ compliance to the treatment (vs $87 \%$ for the whole population) but that the difference was not significant, probably due to the small sample of patients. No direct influence of gender, neither the distance from the apheresis centre or the age at initiation were confirmed as determinants of low compliance. Also, the presence of cardiovascular disease in patients or siblings was not a factor influencing the compliance of the treatment. Genotype did not predict LA compliance, although the least compliant patients were those with hoFH undergoing weekly LA. Genotype was, however, confirmed as a predicting factor of TC, LDL-c, HDL-c and Lp(a) and a significant difference in lipid levels before LA was observed between hoFH and heFH patients (data not shown). This does not imply that a decrease in the frequency of LA would be beneficial for these patients: those who had more than 2 LA procedures per month were the most severe phenotypes, so an even higher frequency would be appropriate and adequate to address the severe condition. Also, the harm that may derive from a missed treatment has to be put in relation with the total number of procedures: the higher the frequency of LA the lower the impact of one missed procedure.

The number of missed procedures was surprisingly low: over 18 years of follow-up, as high as $87.5 \%$ of all prescribed procedures had been performed. This result was rather unexpected because LA patients have no apparent symptoms in comparison with dialysis patients and therefore we had thought that compliance would have been much lower. Patients treated by LA suffer from chronic disease and are likely to be non-compliant. Surprisingly, there is no data assessing compliance 
or adherence with this prescribed procedure in such populations. Furthermore, assessing adherence is of major importance when discussing the number of procedures to be prescribed in a single patient. Poor adherence is considered a critical barrier to treatment success and premature discontinuation in such treatment may have dramatic consequences. This has been confirmed in several studies assessing compliance with drug treatment for CVD prevention [20,21]. As low adherence has been associated with increased cardiovascular outcomes, we would expect a low progression of CVD and CV mortality in our cohort.

Over the past 20 years, long-term follow-up of these patients revealed that, when apheresis is performed regularly, levels of LDL decreased to levels that cannot be obtained with drug therapy alone. Nonetheless, despite regular LA, a significant proportion of severe $\mathrm{FH}$ patients do not reach treatment goals or continue to develop significant atherosclerotic disease and consequently remain at increased risk for coronary events. We have recently shown that a residual risk of CVD is present in patients despite the ongoing intensive treatment: in a cohort of 53 patients treated with LA over maximally tolerated pharmacological treatment, a $100 \mathrm{mmol} / \mathrm{L}$ increase in cumulative total cholesterol was associated with a 2-fold increased risk of a cardiovascular event [8]. One of the main obstacles to lowering lifelong cholesterol exposure in LA treated patients has been the LDL-C rebound. Combination therapy of LA with statins and ezetimibe progressively reduced the magnitude of the post-apheresis rebound in LDL-C levels, although the type of LDL-R mutation significantly contributes to LDL-C rebound [22]. The reduction of other risk parameters such as other atherogenic lipoproteins (Lp(a), VLDL, TG), which are considered independent risk factors and act on inflammatory parameters (fibrinogen, C-reactive protein, blood viscosity ...), may have a complementary benefit and demand attention [23].

A residual risk is therefore still present in $\mathrm{FH}$ patients despite maximal therapy and these patients emphasize the need for intensive lipid-lowering regimen and new treatment options to further decrease LDL-C levels in order to provide superior benefits [24]. The recent commercialization of new therapies for hypercholesterolemia is very promising for a further reduction in CV mortality and optimization of cholesterol burden in FH patients [25,26]. A significant reduction in the frequency of LA treatment has been recently observed after the introduction of PCSK9-inhibitors in HeFH patients: 26 patients out of 41 treated by alirocumab could completely suspend LA, while overall 92.7\% patients halved the LA frequency [27].

This study has several limitations. First, the number of patients was small as a result of the limiting criteria for LA. Consequently, results on adherence do probably reflect a selection bias: the acceptance of LA treatment itself represents an initial step towards higher adherence. Second, the reasons for non-attendance were given by the patients. We therefore cannot exclude the possibility that non-compliers provided a medical or non-medical explanation in order to justify a missed procedure. Third, we were not able to clearly identify whether non-compliers had a worse clinical outcome; such an analysis would be difficult because some patients might have stopped their treatment as a result of an event. Furthermore, the age at the beginning of treatment was not standardised. The number of patients treated with this combination is small and does not achieve the power and sample size required for a randomized controlled trial. Due to the severity of the disease, it is not ethical to perform randomized trials in FH patients.

In conclusion, this is the first study investigating the compliance of LA treatment which appears to be satisfactory in our cohort. The only parameter associated with low compliance was the frequency of the procedure. The good compliance observed in this study should be analysed in other groups in order to assess whether our results are generalizable.

LA in combination with drug therapy effectively reduces LDL-C levels. Together with all current available treatments, it might finally allow these patients to reach therapeutic LDL-C objectives, so as to reduce the residual risk of CVD along with LDL-C levels both in primary and in secondary prevention.

\section{Conflicts of interest}

SB declares having received honoraria from Amgen, Sanofi; AG declares having received honoraria from AMGEN, Sanofi and Novartis; AC has received honoraria from Amgen SAS and Alexion Pharma France SAS, and is currently receiving a grant from Alexion Pharma France SAS; MJC declares having received honoraria from Amgen, MSD, Sanofi and Regeneron, Servier and Unilever; EB declares having received honoraria from AstraZeneca, AMGEN, Genfit, MSD, Sanofi and Regeneron, Unilever, Danone, Aegerion, Chiesi, Rottapharm-MEDA, Lilly, Ionis-pharmaceuticals; SS declares having received honoraria from Octapharma, Alexion, Terumo-bct, Agerion phama, Sanofi; Remaining authors have nothing to disclose.

\section{Author contributions}

All authors contributed to conception, design, drafting and final revision and approval of the article. SB, AG, ED, SS provided to data collection and analysis; AC performed genetic analysis; RB provided lipid and biochemical profiling.

\section{Acknowledgements}

Chronic diseases require an important development of the "treatingtreated" relation, and we would like to thank all the nurses and nonmedical and medical staff of the LA centre who have been devoted to patient care for several years.

\section{References}

[1] M.S. Brown, J.L. Goldstein, Expression of the familial hypercholesterolemia gene in heterozygotes: mechanism for a dominant disorder in man, Science 185 (4145) (1974) 61-63.

[2] J.L. Goldstein, H.H. Hobbs, M.S. Brown, Familial Hypercholesterolemia, in: C.R. Scriver, et al. (Ed.), The Metabolic Basis of Inherited Disease, 7 th Ed, McGraw Hill, New York, 1995, pp. 1981-2030.

[3] M. Benn, G.F. Watts, A. Tybjærg-Hansen, B.G. Nordestgaard, Mutations causative of familial hypercholesterolaemia: screening of 98098 individuals from the Copenhagen General Population Study estimated a prevalence of 1 in 217, Eur. Heart J. 37 (17) (2016 May 1) 1384-1394, https://doi.org/10.1093/eurheartj/ ehw028.

[4] R. Do, N.O. Stitziel, H.H. Won, A.B. Jørgensen, S. Duga, et al., Exome sequencing identifies rare LDLR and APOA5 alleles conferring risk for myocardial infarction, Nature 518 (7537) (2015 Feb 5) 102-106, https://doi.org/10.1038/nature13917.

[5] L. Mundal, M. Sarancic, L. Ose, P.O. Iversen, J.K. Borgan, et al., Mortality among patients with familial hypercholesterolemia: a registry-based study in Norway, 1992-2010, J. Am. Heart Assoc. 3 (6) (2014 Dec 2) e001236, , https://doi.org/10. 1161/JAHA.114.001236.

[6] A.D. Marais, Familial hypercholesterolaemia, Clin. Biochem. Rev. 25 (1) (2004) 49-68.

[7] M. Cuchel, E. Bruckert, H.N. Ginsberg, F.J. Raal, R.D. Santos, et al., Homozygous familial hypercholesterolaemia: new insights and guidance for clinicians to improve detection and clinical management. A position paper from the Consensus Panel on Familial Hypercholesterolaemia of the European Atherosclerosis Society, Eur. Heart J. 35 (32) (2014 Aug 21) 2146-2157, https://doi.org/10.1093/eurheartj/ehu274.

[8] E. Bruckert, O. Kalmykova, R. Bittar, V. Carreau, S. Béliard, et al., Long-term outcome in 53 patients with homozygous familial hypercholesterolaemia in a single centre in France, Atherosclerosis 257 (2017) 130-137, https://doi.org/10.1016/j. atherosclerosis.2017.01.015.

[9] G.R. Thompson, R. Lowenthal, N.B. Myant, Plasma exchange in the management of homozygous familial hypercholesterolaemia, Lancet 1 (7918) (1975 May 31) 1208-1211.

[10] W. Stoffel, H. Borberg, V. Greve, Application of specific extracorporeal removal of low density lipoprotein in familial hypercholesterolaemia, Lancet 2 (8254) (1981 Nov 7) 1005-1007.

[11] G.R. Thompson, M. Barbir, D. Davies, P. Dobral, M. Gesinde, et al., Recommendations for the use of LDL apheresis, Atherosclerosis 98 (2008) 247-255, https://doi.org/10.1007/8904_2011_45.

[12] M. Harada-Shiba, H. Arai, S. Oikawa, T. Ohta, T. Okada, et al., Guidelines for the management of familial hypercholesterolemia, J. Atherosclerosis Thromb. 19 (2012) 1043-1060.

[13] A.C. Goldberg, P.N. Hopkins, P.P. Toth, C.M. Ballantyne, D.J. Rader, et al., Familial 
hypercholesterolemia: screening, diagnosis and management of pediatric and adult patients: clinical guidance from the National Lipid Association Expert Panel on Familial Hypercholesterolemia, J. Clin. Lipidol. 5 (3 Suppl) (2011 Jun) S1-S8, https://doi.org/10.1016/j.jacl.2011.04.003.

[14] G.R. Thompson, A. Catapano, S. Saheb, M. Atassi-Dumont, M. Barbir, et al., Severe hypercholesterolaemia: therapeutic goals and eligibility criteria for LDL apheresis in Europe, Curr. Opin. Lipidol. 21 (6) (2010 Dec) 492-498, https://doi.org/10 1097/MOL.0b013e3283402f53.

[15] A.A. Kroon, M.A. van't Hof, P.N. Demacker, A.F. Stalenhoef, The rebound of lipoproteins after LDL-apheresis. Kinetics and estimation of mean lipoprotein levels, Atherosclerosis 152 (2) (2000 Oct) 519-526, https://doi.org/10.1016/S00219150(00)00371-3.

[16] B.R. Gordon, S.F. Kelsey, P.C. Dau, et al., Liposorber Study Group, Long-term effects of low-density lipoprotein apheresis using an automated dextran sulphate cellulose adsorption system, Am. J. Cardiol. 81 (1998) 407-411.

[17] A. Krebs, K. Krebs, F. Keller, Retrospective comparison of 5 different methods for long-term LDL-apheresis in 20 patients between 1986 and 2001, Int. J. Artif. Organs 27 (2004) 137-148.

[18] R. Bambauer, R. Schiel, R. Latza, Low density lipoprotein apheresis in treatment of hyperlipidemia: experience with four different technologies, Ther. Apher. 4 (3) (2000 Jun) 213-217.

[19] C. Keller, P. Grutzmacher, F. Bahr, A. Schwarzbeck, A.A. Kroon, A. Kiral, LDLapheresis with dextran sulphate and anaphylactoid reaction to ACE inhibitors [letter], Lancet 341 (1993) 60-61.

[20] J.N. Rasmussen, A. Chong, D.A. Alter, Relationship between adherence to evidence- based pharmacotherapy and long-term mortality after acute myocardial infarction, J. Am. Med. Assoc. 297 (2007) 177-186.

[21] S.H. Simpson, D.T. Eurich, S.R. Majumdar, et al., A meta-analysis of the association between adherence to drug therapy and mortality, BMJ 333 (2006) 15.

[22] J.P. Drouin-Chartier, A.J. Tremblay, J. Bergeron, B. Lamarche, P. Couture, The lowdensity lipoprotein receptor genotype is a significant determinant of the rebound in low-density lipoprotein cholesterol concentration after lipoprotein apheresis among patients with homozygous familial hypercholesterolemia, Circulation 136 (2017) 880-882, https://doi.org/10.1161/CIRCULATIONAHA.117.029435.

[23] B.S. Sachais, J. Katz, J. Ross, D.J. Rader, Long-term effects of LDL apheresis in patients with severe hypercholesterolemia, Clin. Apher. 20 (4) (2005 Dec) 252-255, https://doi.org/10.1002/jca.20036.

[24] M. Farnier, E. Bruckert, Severe familial hypercholesterolaemia: current and future management, Arch. Cardiovasc Dis. 105 (12) (2012 Dec 6) 656-665, https://doi. org/10.1016/j.acvd.2012.05.011 Epub 2012 Oct 6.

[25] M.S. Sabatine, R.P. Giugliano, A.C. Keech, N. Honarpour, S.D. Wiviott, et al., Evolocumab and clinical outcomes in patients with cardiovascular disease, N. Engl. J. Med. 376 (2017) 1713-1722, https://doi.org/10.1056/NEJMoa1615664.

[26] J.G. Robinson, M. Farnier, M. Krempf, J. Bergeron, G. Luc, et al., Efficacy and safety of alirocumab in reducing lipids and cardiovascular events, N. Engl. J. Med. 372 (2015) 1489-1499.

[27] P.M. Moriarty, K.G. Parhofer, S.P. Babirak, M.A. Cornier, P. Barton Duell, et al., Alirocumab in patients with heterozygous familial hypercholesterolaemia undergoing lipoprotein apheresis: the ODYSSEY ESCAPE trial, Eur. Heart J. 37 (2016) 3588-3595, https://doi.org/10.1056/NEJMoa1501031. 